\title{
Supporting Simulations to Guide Engineering Design
}

\author{
Mark S. Shephard \\ Scientific Computation Research Center, Rensselaer Polytechnic Institute, Troy, NY 12810 \\ Mark W. Beall, Bruce E. Webster \\ Simmetrix, Inc., 10 Halfmoon Executive Park Drive, Clifton Park, NY 12065
}

\section{Introduction}

Although computer-aided engineering (CAE) technologies are a critical component to the engineering design process, their effective integration into design processes continues to be awkward. This has been particularly true of more advanced simulation technologies such as finite element analysis. Developments over the past decade on technologies like the automatic generation of finite element mesh, and other analysis discretizations, directly from solid model representations $[1,8]$ provide one of the tools to allow the effective use of simulation during design. However, a number of additional capabilities and structures are needed to truly integrate the simulation technologies into engineering design.

One of the needed capabilities would support the effective association of simulation information to evolving design definitions. Although the methods used to automate mesh generation of solid models provides a means to link the simulation results model's boundary representation, it is well recognized such representations are not the most appropriate design representations. A second needed capability is the adapt control of automated analysis model construction methods that accounts for the current state of the design and provides a means to control the simulation accuracy needed for the design performance parameters the simulation is to provide. This paper will discuss efforts on the development of a set of structures and methods that when combined with available advanced CAD representations and CAE tools will support the effective application of simulation technologies in support of engineering design processes.

The next section gives a brief overviews of the component technologies needed to support the concept of simulation-based design where simulation can automatically be applied to provide the product performance evaluations, design parameter sensitivity information and verification. Section 3 provides additional information on the key component of the simulation model manages which interacts directly with the highest level of design definition. Section 4 briefly indicates how these technologies are beginning to be used in the automotive industry to support advanced design and virtual prototyping.

\section{Simulation-Based Design System Components}

Figure 1 provides an overall view of the functional components needed to support simulationbased design (SBD). These components include the product data managers, computer-aided design tools and computer-aided engineering tools heavily used in industry. It also includes four new components (within the dashed box) that are currently under development to support the integration of adaptive simulations into the design process. The interactions indicated by the arrows in Figure 1 represent only those interactions activated when an engineering simulation is executed. The function of the existing components and their interaction are summarized as:

- The Product Data Manager initiates requests to have engineering performance parameters evaluated for a given design. 


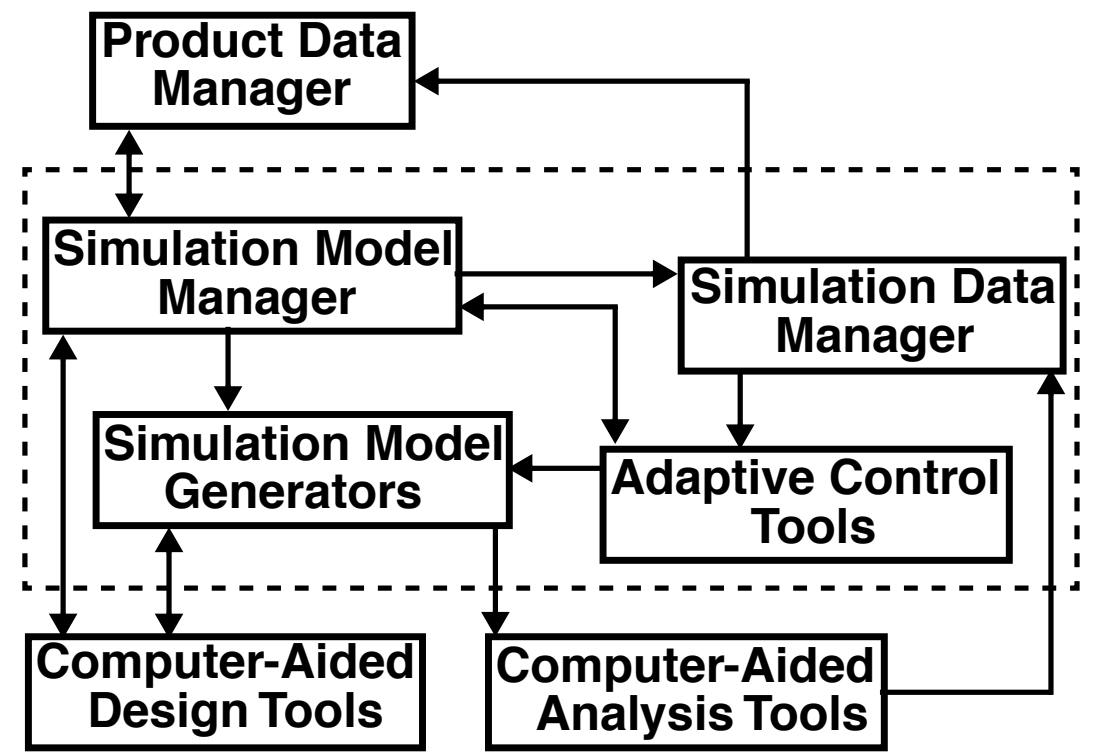

Figure 1. Functional components needed for simulation-based design with the four new components currently under development within the dashed box.

- The Computer-Aided Design (CAD) Tools house the design models and support interactions with those models. Today's commercial CAD systems include a solid model defined in terms of a boundary representation. They also often include some form of feature model that can be used in support of the design process.

- The Computer-Aided Engineering (CAE) Tools include the engineering analysis programs in use today (e.g., 1-D engineering performance models, finite element analysis, etc.). The key characteristic of the CAE tools is that they operate on a model specific to the analysis method being used which is not necessarily maintained as a part of the design representation. When used in the appropriate manner, the CAE tools can compute the raw information needed to answer the given performance request.

The four new components being developed to support simulation-based design are [10]:

- The Simulation Model Manager that is responsible for controlling the overall simulation process and providing the base simulation model definition information needed by the other components.

- The Simulation Data Manager that is responsible for housing the simulation result information as needed during the simulation process.

- The Adaptive Control Tools that are responsible for selecting and controlling the simulation models so that the estimates of the performance parameters are to the level of accuracy requested.

- The Simulation Model Generators that are responsible for constructing the models used by the CAE tools accounting for the current design information and adaptively defined analysis model construction information.

Within a simulation-based design process, the product data manager communicates its request for performance parameter evaluation to the simulation model manager. The simulation data manager communicates information about the processes performed back to the product data manager. The simulation model manager executes two way communications with the computer-aided design tools. It provides the simulation model generators with information needed in constructing the input to the CAE tools, and provides the simulation data manager information needed to evaluate 
the requested performance parameters based on the CAE simulation results. The simulation model manager interacts with the CAD tools to ensure that a detailed geometric model representation consistent with the component model definition appropriate for the simulation models needed is available.

The two way communications between the simulation model manager and adaptive control tools are used to communicate information on the performance information requested and the required model information and information on additional model change information that is required in the process of constructing the simulation models. The adaptive control tools communicate with the simulation model managers to indicate the simulation models that need to be generated. The adaptive nature of the simulation control process requires communication with the simulation data manager to obtain the results information needed to determine modifications needed in the simulation model for the next analysis iteration.

The simulation model generators obtain the information on which simulation models are to be constructed from the adaptive control procedures. To construct the simulation models, information is needed from the simulation model manager. In addition, since in many cases the model generation procedures need detailed geometric information, this information is obtained from an interaction with the solid modeler. This interaction is indicated as two-way since the existence of an appropriate boundary representation and operator libraries for the detailed geometric domain models exist such that the simulation model generation tools can obtain all needed domain information through simple interrogations of the geometric model. The simulation model manager is responsible for providing the information needed by the CAE tools in the form they require.

The CAE tools provide the simulation results information to the simulation data manager which is capable of extracting the results information needed to control the adaptive control processes or to evaluate the requested performance parameters in a form needed by the product data management system.

\section{Simulation Model Manager}

Given a request to determine one or more performance parameters to a given level of accuracy, the simulation model manager must determine if the state of the design has evolved to the point that the simulations required for those evaluations can be done, and, if it has, to provide the simulation procedures with the design definition information needed to perform the simulations. The Simulation Model Manager (SMM) is the key functional component that links simulation technologies into the design process.

To support simulation processes a high level functional view of the design is needed. This model must include all functional components that contribute to the requested simulations. The individual components need to be attributed with the additional information to support the operations to be executed during the required simulations. Information important to simulation-based design includes the appropriate level of geometry definition, material properties, loadings, and boundary conditions associated with the component and its functional interfaces to other components. An examination of commercial CAD systems indicates that structures needed to support aspects of the functionalities and structures are available since these systems typically provide:

- An graph of components in an assembly model defining the instances of the individual components with linkages providing a means to provide information on their interactions [6].

- The ability to define the geometry and obtain a boundary representation [11] of assembly model components for which its geometry needs to be defined at this point in the design.

- An ability to associate information with the components of the assembly graph. 
Although it is possible, with appropriate care, to obtain the information needed to support simulations from the CAD system, the direct use of these structures does not effectively support the execution of adaptive simulation processes. In addition, there is limited uniformity between the CAD systems with respect to how one interacts with the structures. On the other hand, it is possible to add the structure needed for the simulation model manager to effectively integrate with the CAD system tools at the assembly tree level. In addition, the most technically complex aspect of interacting with the CAD model, the interrogation and modification of the detailed geometric model, is effectively supported by the CAD systems in a fairly uniform manner.

To support the high level definition of the design for simulation processes, a component model must be defined. From the simplest point of view, the component model consists of a set of components and interfaces between the components where the interfaces are functionally meaningful. To meet the needs of SBD, each component can be defined in terms of sub-components providing a hierarchal description of the model. Components can have a rich set of attributes associated with them and the propagation of the attributes associated with components within the hierarchy is governed by a set of rules. In addition, it must be possible to have a complete geometric representation of the component model generated using the functionality of the CAD tools.

As a simple example of how the component model is defined, consider a heat exchanger design. The component model for this is shown in Figure 2. The figure on the left shows the coarsest component representation where the heat exchanger unit connects to the inlet and outlet duct. In the middle picture, it can be seen that the heat exchanger unit is comprised of a core, fan and filter. Note that the relation between the heat exchanger and the inlet is actually between the fan and the inlet duct, the ability to have these hierarchical relationships is part of the power of the component model representation. The picture on the right shows that the core has a further decomposition.
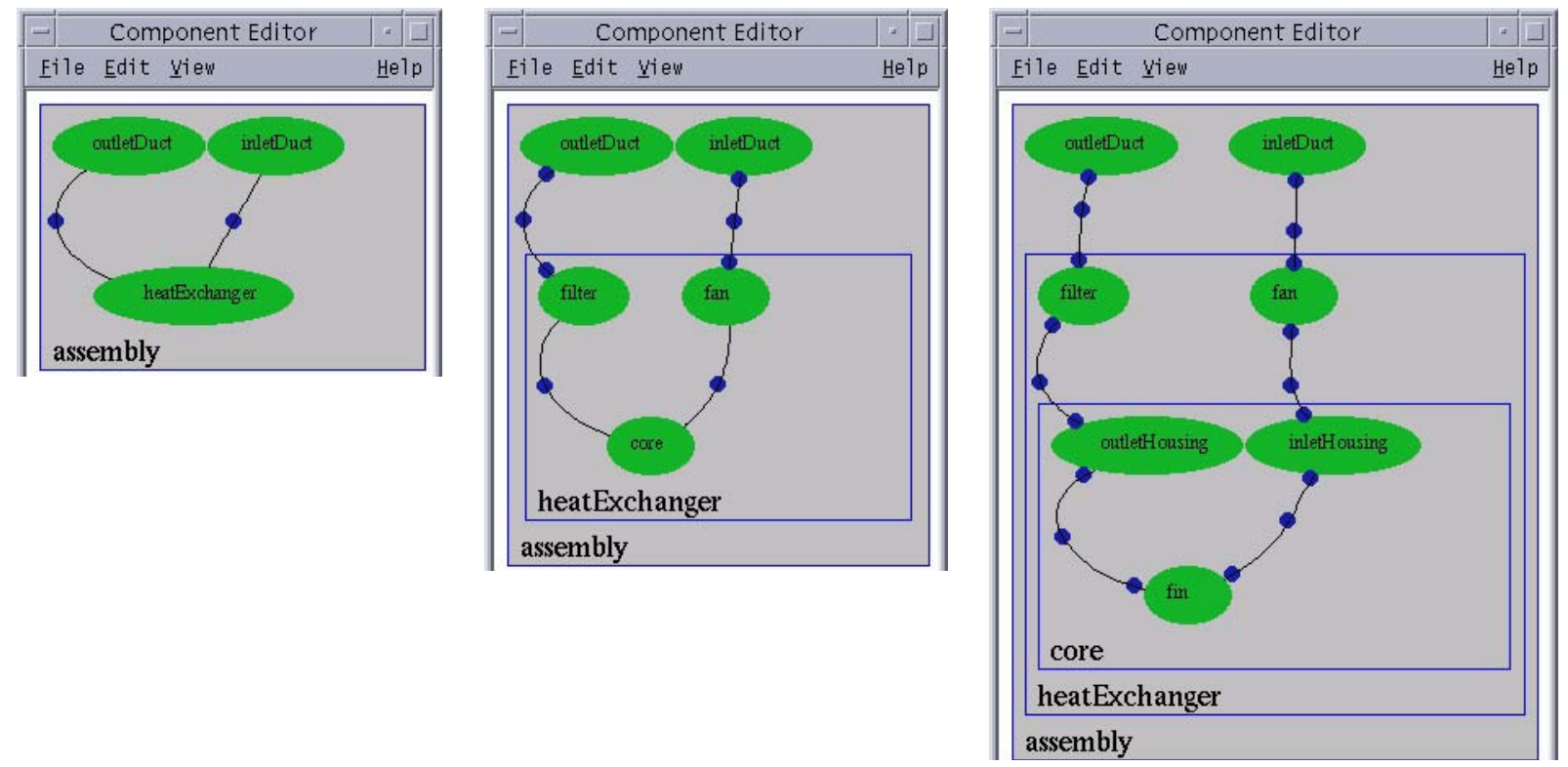

Figure 2. Heat exchanger component model.

From the conceptual level, the instance of the component model for a specific simulation is constructed from the master model assembly (also called feature) model $[2,4]$ taking into account the functional components to be included for the simulation process. Methods for extracting the component models accounting for multiple viewpoints are an active area of research $[3,4,5]$ which has 
yet to be formalized to the point that the CAD systems fully support these concepts. However, it is not difficult to use the structures within the SMM to support the use of these concepts to the level needed to support adaptive simulation processes.

Within a simulation-based design process, attributes are defined as any information which is associated with a component that is not part of the geometric model definition needed to support the simulation processes and include:

- Physical attributes of loads, material properties and boundary conditions.

- Modeling attributes that indicate the appropriate mathematical models and specific model idealization including geometric simplifications.

- Numerical analysis attributes used by the procedures defining the input needed to the CAE analysis procedures.

- Engineering performance parameters, the evaluation of which is the goal of the simulations.

Although the component model can deal with the association of attributes to the model components and interfaces, a richer functionality is needed to define the physical attributes which are tensors that have general dependencies and variations [7].

The last key structure within the SMM is the topology associated with the geometric model. Since the geometric models needed for simulation processes are often general combinations of solids, surfaces, curves and points, a complete non-manifold representation in the form of the radial-edge data structure [11] is used. The primary reason the simulation model manager needs to maintain the topology of the geometric model is that CAD systems are not uniform in the way they treat topology, including some not automatically determining and representing specific non-manifold situations. However, since the geometric modelers, when properly used, can evaluate the nonmanifold situations and support the geometric interrogations needed in the simulation processes, the geometric modeling functionality required can be supported by the combination of a nonmanifold topology with the CAD system solid modeler. Within the simulation model manager, all interrogations of the geometric domain are made with operators that are driven by entities in the non-manifold topology. These operators are constructed for the various CAD system geometric modelers using their operator libraries $[1,8,9]$.

For many classes of simulation, the analysis model is constructed from a spatial discretization of the geometric model. In these cases the relationship of the component model entities to the geometric model entities and the process of transforming attributes defined on the component model to the geometric models entities are critical. The CAD system assembly modelers maintain the relationship between the entities in the assembly model and the entities in the boundary representation of the geometric model it maintains. Therefore, the basic information the Simulation Model Manager needs to construct these relationships for the viewpoint specific component models is available. Proper care must be taken to construct the correct representations of any physical interfaces that may be defined by the interaction of component model entities.

The implementation of these capabilities in the SSM is supported by class hierarchy that links the CAD system assembly components, the CAD system solid models with the SMM components of the simulation model assembly graph, the simulation attributes, and the non-manifold topology of the appropriate solid model instance. The SMM components house the appropriate links to the adaptive simulation control procedures and the associated simulation models.

Figure 3 gives a simple example of the power of being able to control SBD processes through a component model that links to the detailed geometry needed for a simulation process. The three components of the heat exchange system are the same in both cases. The only difference is in the 
exchange unit subcomponents in the two cases. For the case on the right there are three instances of a subcomponent with an elongated cross section correctly positioned while in the figure on the left there are a number of instances of a circular tube cross section correctly positioned. The attributes as needed for a flow simulation are the same for both cases and the combination of the Simulation Model Manager and CAD system geometric modeler can provide the appropriate information to the automate mesh generators and CAE flow solver even though the actual geometry is quite different in the two cases.
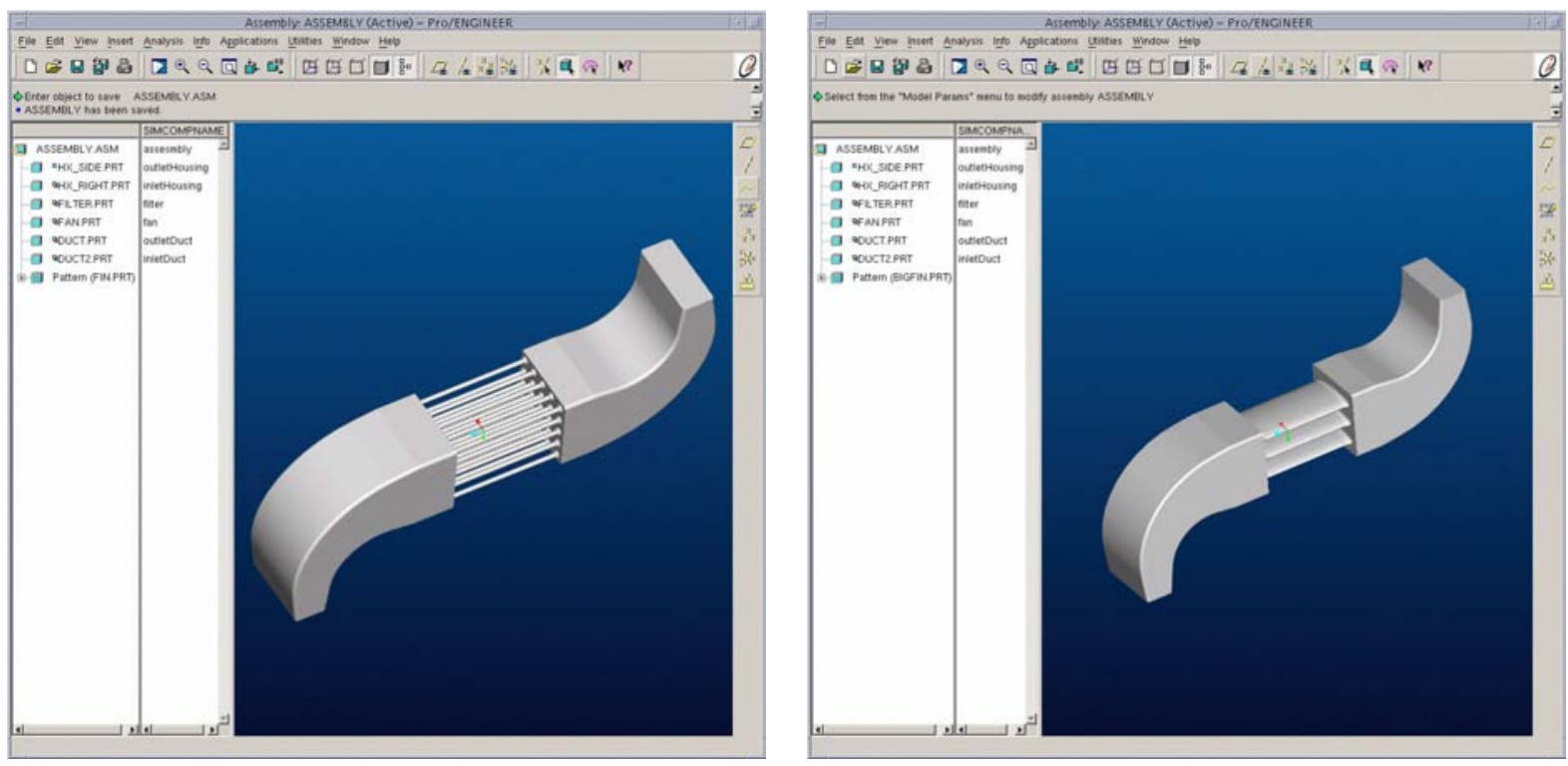

Figure 3. Two heat exchanger designs.

\section{Application to Automotive Climate Control Systems Design}

The definition and construction of the four new functional components needed to support simulation-based design is being guided by the experience and knowledge being gained through the development of multiple simulation-based design systems working directly with industry. The most mature of these systems has been developed as a collaborative effort with a tier one automotive supplier. The specific system is focused on the design of automotive climate control systems and takes advantage of previous efforts to define a parametric vehicle model in the Pro/E CAD system. This parametric model includes a thermal/fluids engineering viewpoint structured to support accurate and validated simulation processes as needed to support the design of automotive climate control systems [10].

The users of the SBD system are design engineers who are charged with the selection and sizing of the climate control components associated with a vehicle platform to most effectively satisfy a set of design goals and constraints. The design goals and constraints range from the geometric parameters of climate control, to overall passenger comfort under a variety of driving conditions.

The parametric vehicle model has been decomposed into a set of high level functional components so that it can be synchronized with the component model in the simulation model manager. Figure 4 shows a generic view of some of the major functional components for a particular vehicle platform. As part of the process the designer is able to select from and define the major com- 


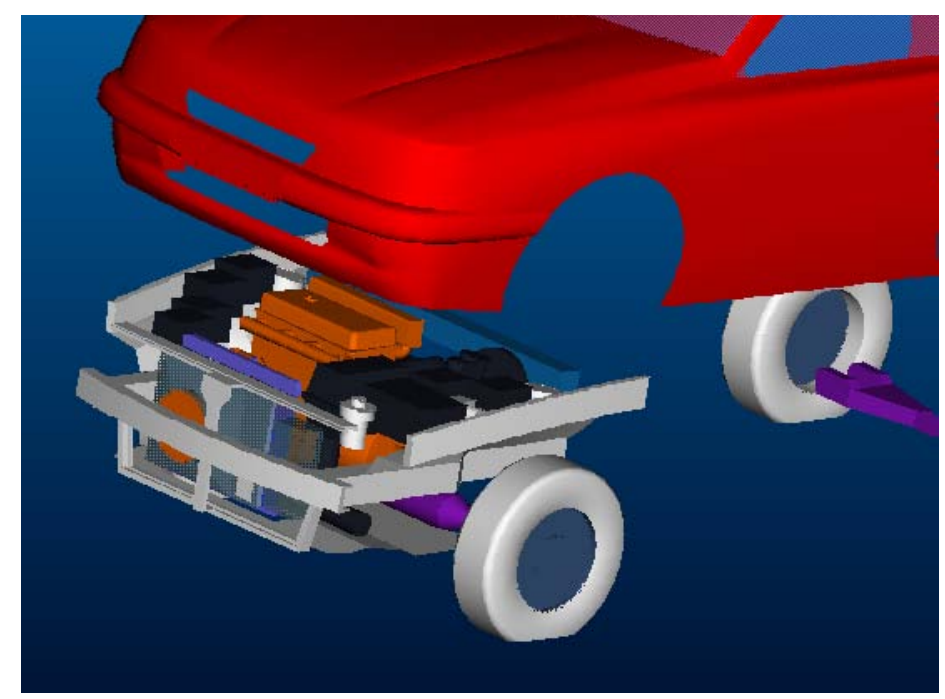

Figure 4. Exploded view of major functional components.

ponents as configurations of subcomponents that are typically selected from libraries of available parts for items such as fan blades, pumps, etc.

The designer works with high level performance parameter specifications which in the case of interior comfort control design are carefully qualified by SBD specialists with respect to the comfort level response of occupants placed in the vehicle. Although performance parameters are typically related to a simple physical parameter, their evaluation often require the execution of extensive coupled simulations. Such an example is radiator top water temperature that must be predicted to within a few degrees $\mathrm{C}$. The prediction of this one parameter requires full exterior/ underhood transient flow analysis of complete drive cycles, coupled with calibrated engineering models for the heating/cooling system and solar radiation.

The determination of specified performance parameters begins from a simulation template that is constructed from a priori knowledge codified into the analysis control attributes about what methods are appropriate at this point in the design process. The modeling and numerical analysis attributes associated with the components are used to provide the needed information which must include the levels of model idealization possible for each component as well as how various components can interact.

In those cases where generalized numerical analysis procedures are used, a spatial discretization of the domain in terms of a mesh is needed. To support the mesh generation process the non-manifold topology and links to the geometric information in the CAD system is used. This process requires specific interactions between SEED component, attribute and non-manifold model topology and the Pro/E assembly tree and geometric modeling engine. In cases where analysis idealizations reduce the dimensionality or simplify portions of the geometric domain, the system contains sufficient information to execute those processes. The initial numerical analysis attributes contain information that indicates the initial mesh size field over the domain and parameters to appropriately control the execution of the CAE program.

Although the application of adaptive model and discretization selection is the most appropriate means to control the accuracy of the solution process, reliable means for the a posteriori prediction of the solution error and adaptive control methods for all error contributions are not available. In addition, in many design situations, the similarity of the designs and analyses performed on 
them is such that capturing validated expert analysis models and procedures is a highly effective means of ensuring simulation reliability. The current SBD application does contain adaptive methods for controlling mesh discretization for 3-D fluid flow analyses over complex geometries. For other analyses, and for the creation of effective initial meshes, a substantial effort was expended on capturing the knowledge of CAE experts gained through the execution of many analyses over a range of design configurations for which validation was performed.

The SBD application has codified the analyses processes for a variety of virtual tests that are performed using well controlled thermal/flow simulations. Many of these simulations require the transient solution of the Navier Strokes equations over general domains where thousands of time steps must be solved over meshes with millions of mesh points. The flow results in the underhood region critical to this process are shown in Figure 5. The results obtained using these procedures have been compared to wind tunnel experimental tests for integrated air flow prediction and are found to be within the levels of accuracy needed in the design process.

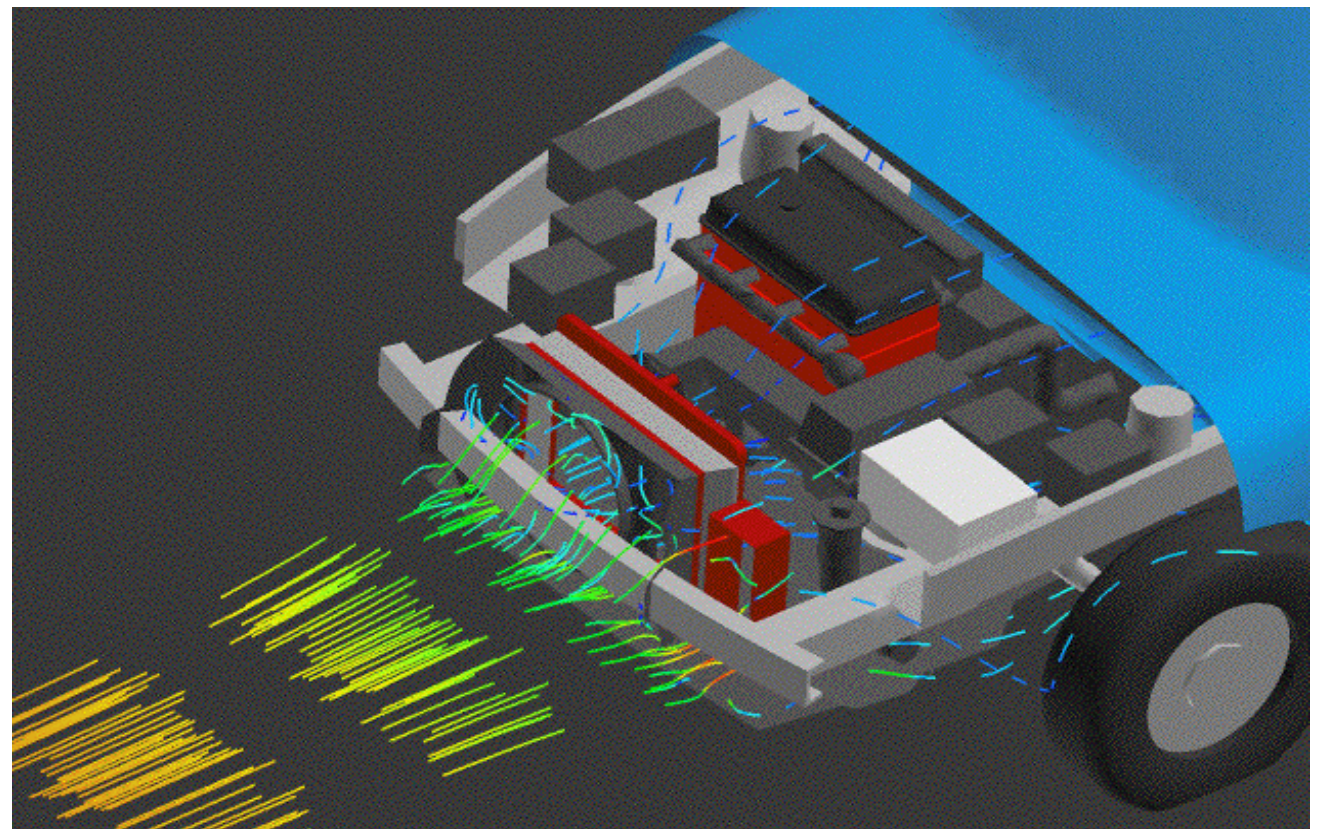

Figure 5. Flow results in the critical under the hood region.

\section{Closing Remarks}

This paper has introduced a set of functional components that when combined with existing CAD and CAE technologies can support the effective application of simulation in engineering design. The role of the Simulation Model Manager and its interaction with the product design definition has been discussed. The Simulation Model Manager supports the execution of advanced engineering simulations for the accurate prediction of performance parameters critical to design evaluation. Since the Simulation Model Manager maintains an understanding of the high level design components, the evaluation of parameters for response surface construction and determination of design sensitivities for parameters component sizing or shape parameter optimization should be reasonably straight forward capabilities to add. 


\section{Acknowledgment}

The authors gratefully acknowledge the NIST ATP program, award number 70NANB1H3035, for supporting the development of SEED.

\section{References}

[1] Beall, M.W., Walsh, J. and Shephard, M.S., "A comparison of techniques for geometry access related to mesh generation" to appear, Engineering with Computers, 2004.

[2] Bidarra, R. and Bronsvoort, W.F., "Semantic feature modeling", Computer-Aided Design, 32:201-225, 2000.

[3] Bronsvoort, W.F. and Jansen, F.W., "Feature modeling and conversion - key concepts to concurrent engineering", Computers in Industry, 21(1):61-86, 1993.

[4] Hoffmann, C.M. and Joan-Arinyo, R., "CAD and the product master model", ComputerAided Design, 30(11):905-918, 1998.

[5] Hoffmann, C.M. and Joan-Arinyo, R., "Distributed maintenance of multiple project views", Computer-Aided Design, 32:421-431, 2000.

[6] Noort, A., Hoek, G.F.M. and Bronsvoort, W.F., "Integrated part and assembly modeling", Computer-Aided Design, 34:899-912, 2002.

[7] O'Bara, R.M., Beall, M.W. and Shephard, M.S. "Attribute Management System for Engineering Analysis", Engineering with Computers, 18(4):339-351, 2002.

[8] Shephard, M.S., "Meshing environment for geometry-based analysis", International Journal for Numerical Methods in Engineering, 47(1-3):2000.

[9] Shephard, M.S. and Georges, M.K., "Reliability of Automatic 3-D Mesh Generation", Comp. Meth. Applied Mechanics and Engng., 101, pp. 443-462, 1992.

[10] Shephard, M.S., Beall, M.W., O'Bara, R.M. and Webster, B.E., "Toward simulation-based Design" to appear, Finite Elements in Analysis and Design, 2004.

[11] Weiler, K., "The Radial Edge Structure: A Topological Representation for Non-Manifold Geometric Modeling”, Geometric Modeling for CAD Applications (IFIP WG5.2 Working Conference Rensselaerville, N.Y., May 12-14, 1986), Wozny, M. J., McLaughlin, H., Encarnacao J., Eds., 1988 pp. 3-36. 